\title{
HYPERNATRAEMIC DEHYDRATION IN INFANTILE GASTRO-ENTERITIS
}

\author{
BY \\ DUNCAN MACAULAY and MARGARET I. BLACKHALL \\ From the Duchess of York Hospital for Babies, Manchester
}

(RECEIVED FOR PUBLICATION DECEMBER 19, 1960)

\begin{abstract}
Although it is possible to find occasional reports written before the last war of the occurrence of hyperelectrolytaemia in sick infants and children (for early references see Rapoport, 1947) the recognition of the condition as a fairly common one and the elucidation of the clinical features date from 1947 when Rapoport gave an account of 14 patients with hyperosmolarity of the plasma. Of the 14 patients, 10 were suffering from diarrhoea. Five of these were noted to have symptoms of central nervous system disturbance, from marked lethargy to convulsions. The first indication of the possible frequency of this state came in a report by Tarail, Bass and Runco (1953) who found that, of 191 infants with diarrhoea, 21 or $11 \%$ had serum sodium levels of $150 \mathrm{mEq} / \mathrm{litre}$ or higher. In 1955 Finberg and Harrison gave an account of seven years' experience of infantile diarrhoea involving 274 patients. They reported an incidence of hypernatraemia (serum sodium level over $150 \mathrm{mEq} / 1$.) of $25 \%$ and pointed out the great frequency of 'manifestations referable to the nervous system', nearly two-thirds of the hypernatraemic cases showing such signs. They reported, for the first time, the occurrence of raised protein concentrations in the cerebrospinal fluid, and drew attention to the fact that nearly half the cases did not show the customary signs of dehydration. They indicated the danger that rapid rehydration with dilute fluids might give rise to symptoms similar to those seen in water intoxication, especially to convulsions, and they suggested that hypernatraemia could be a cause of permanent brain damage. This paper seemed to define a distinct clinical entity of considerable significance. It was followed by one by Weil and Wallace in 1956 which emphasized the points made by Finberg and Harrison, especially the neurological disturbances. Several other papers from America have reported similar findings and opinion in that country is probably represented by the account of 'Hypertonic dehydration' given by Cooke (1959). According to this writer hypertonic
\end{abstract}

dehydration occurs in approximately $20 \%$ of cases of diarrhoea in infancy; 'of particular importance are the cerebral changes which result from severe hyperosmolarity'; the development of oedema during treatment is referred to as 'characteristic'.

From consideration of the available reports the 'clinical picture' of hypertonic dehydration emerges as follows: the patients are predominantly infants in the first year of life; as the result of fluid loss in the stools and by vomiting there is often loss of $10-15 \%$ of the body weight without the classical signs of such a severe degree of dehydration, e.g. the skin turgor may be deceptively normal; symptoms and signs of cerebral damage are common and include disturbances of consciousness ranging from lethargy to coma; hyperirritability to stimuli in spite of extreme lethargy; increased muscle tone amounting in many cases to generalized rigidity with very brisk tendon reflexes; convulsions, especially during rehydration; raised concentration of protein in the cerebrospinal fluid and rather exceptionally, the development of sub-dural effusions. Oedema is liable to appear during the administration of rehydrating fluids, sometimes before full rehydration has been effected. It has been suggested (Franz and Segar, 1959) that this state is becoming commoner and also (Finberg, 1958) that it may be due in many instances to the unwise administration of fluids with 'high-solute' content.

We wish to describe the two cases which first drew our attention to this condition and then to give an account of an analysis of the records of 100 infants with diarrhoea admitted to the Duchess of York Hospital for Babies on whom serumelectrolyte estimations were performed.

\section{Case Reports}

Case 1. A baby girl, 3 months of age, was admitted to hospital with a history of diarrhoea and vomiting for five days. She had been perfectly well before then. On the day of admission, after a vomit, she became pale, 
limp and staring; her breathing was rapid and grunting and she was sent to hospital as '? pneumonia'. On admission she was an ill infant, pale and listless, with a temperature of $102^{\circ} \mathrm{F}$. The skin turgor was considered to be good and the fontanelle was not depressed, but the tongue was dry. The reflexes were brisk. There were a few moist sounds in the right lung. Otherwise examination revealed no abnormalities. Serum electrolyte estimation showed well-marked hypernatraemia and


$\mathrm{mEq} / \mathrm{l}$.). About 24 hours after admission the child was found convulsing. Lumbar puncture was performed. The fluid was slightly blood-stained and had a protein content of $80 \mathrm{mg} . / 100 \mathrm{ml}$. and a high chloride concentration (137 mEq/1.). Serum electrolytes were rechecked at this time and showed persistence of the hypernatraemia (serum $\mathrm{Na} 159 \mathrm{mEq} / \mathrm{l}$.). In view of this, the fluid intake was increased. The following day the baby had another convulsion and she was so drowsy throughout the day that she had to be fed by tube. On the sixth day the fontanelle was noted to be quite full and subdural taps were performed. From the left side $6 \mathrm{ml}$. of blood-stained fluid were obtained and from the right side about $2 \mathrm{ml}$. of turbid fluid. After this there was persistent oozing of fluid from the puncture on the right side for about 24 hours. Thereafter progress was uneventful and the infant was discharged after 17 days in hospital.

Two days after discharge diarrhoea and vomiting recurred and persisted for six days and she was readmitted to hospital. She was again quite a seriously ill child; the temperature was $103 \cdot 4^{\circ} \mathrm{F}$. and she was listless but very jumpy. On this occasion, however, there were marked signs of dehydration; lax skin, dry mouth and sunken fontanelle; after blood for electrolyte estimation was obtained, an intravenous infusion was started. The serum chemistry again showed pronounced hypernatraemia with severe acidosis (serum Na 162, K 6.6, $\mathrm{CO}_{2} 8 \cdot 5, \mathrm{Cl} 128 \mathrm{mEq} / 1$.). Twelve hours after admission the child was convulsing again and anticonvulsants were administered. On the second day the serum sodium was normal and it remained normal on two subsequent examinations. Progress was rapid and she was discharged after 11 days. Just before discharge, when she was clinically and chemically normal, renal function tests were carried out. Urea clearance was $90 \%$ of normal and the output of sodium and chloride in the urine was normal for the estimated intake.

Six weeks after discharge she was seen as an outpatient and apart from mild constipation she seemed well in every way.

Case 2. A 10-week-old baby boy was admitted with a 24-hour history of diarrhoea. He had not vomited but was reluctant to feed and on the morning of the day of admission he had felt cold. Later that day he seemed to have difficulty with breathing and his colour became poor. On admission he was a very ill baby. The temperature was $104^{\circ} \mathrm{F}$.; breathing was rapid and irregular and there was a tinge of cyanosis; he was extremely limp and listless. Hydration was considered to be good; the skin turgor was not appreciably reduced and the fontanelle was not depressed. There were no localizing signs on examination and the diagnosis was obscure. Encephalitis was thought to be a possibility and lumbar puncture was performed. The fluid was slightly turbid from the presence of red blood cells; no organisms were seen on smears and cultures remained sterile. The protein content was $40 \mathrm{mg} . / 100 \mathrm{ml}$. and the chloride concentration very high (162 $\mathrm{mEq} / \mathrm{l}$.). On the morning after admission the infant was still extremely ill and had a high-pitched cry. Fever persisted and a macular rash was present on the trunk. Serum electrolyte examination revealed gross hypernatraemia (serum $\mathrm{Na} 180$, $\mathrm{K} \mathrm{6} \cdot 2, \mathrm{CO}_{2} \mathrm{16} \cdot 7, \mathrm{Cl} 135 \mathrm{mEq} / \mathrm{l}$.). There was a pronounced leucocytosis in the peripheral blood and the urine contained albumen. All other investigations gave normal results. Diarrhoeal stools were passed for a week. On the third day oedema of the legs was noticed; this persisted for nearly a week. In the afternoon of that day he began to convulse. Just before the onset of the convulsion the limbs were stiff and after it he was lethargic and had to be fed by tube. At this stage the blood still showed hypernatraemia (serum $\mathrm{Na} 153$ $\mathrm{mEq} / \mathrm{l}$.). On the fourth day sub-dural taps were done. About $2 \mathrm{ml}$. of blood-stained fluid were obtained from each side. The profound lethargy continued for another two days and then quite quickly the baby began to improve and he was discharged on the fourteenth day apparently well. Two months after discharge he seemed a perfectly normal infant.

These two cases fulfilled many of the criteria laid down by the American workers for this syndrome; they had lost $10 \%$ and $11 \%$ of their body weight (as judged by the weight-gain on rehydration) which puts them in the category of moderately severe dehydration, but, except in the second admission of Case 1, they were not considered to be in need of immediate intravenous fluids. In other words the customary signs of such a degree of dehydration were absent. They showed several of the nervous disturbances mentioned previously, notably profound lethargy, and convulsions during rehydration. Small sub-dural effusions occurred in both patients. In one the C.S.F. protein was high and in the other at the top limit of the normal range for an infant of this age. One of them became oedematous during treatment.

\section{Analysis of Case Records}

Micro methods for serum electrolyte estimation became available at the Duchess of York Hospital for Babies towards the end of 1957 . The case notes of all patients who had this estimation carried out from October 1, 1957, till June 30, 1960, were examined. In this period of two years and nine months, 98 patients with diarrhoea had serum electrolyte estimations performed. For ease of calculation of percentages we have made the figure up to 100 by including the first two cases examined in July 1960 . The series thus consists of 100 consecutive patients admitted to the hospital suffering from diarrhoea on whom figures for serum electrolytes were obtained.

During the period covered by this survey 439 patients were admitted with diarrhoea. Thus, rather less than 


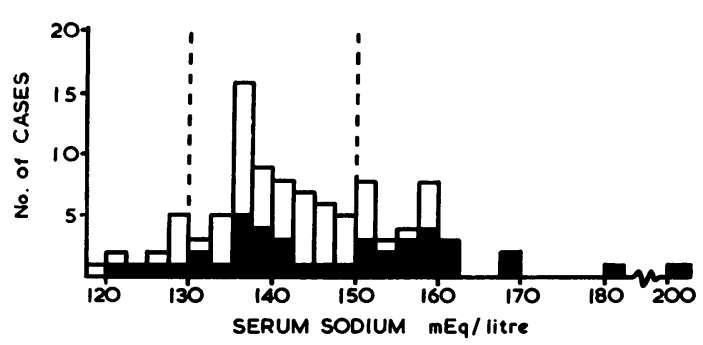

Fig. 1.-Distribution of serum sodium levels at intervals of $2 \cdot 5$ $\mathrm{mEq} / \mathrm{l}$. Vertical dotted lines demarcate hypo-, normo- and hypernatraemia. Cases showing nervous disturbances are indicated in black.

$25 \%$ of them were examined in this way. In general it was the more seriously ill patients who had this examination performed. Because of this selection care must be exercised in making general statements about infants with diarrhoea.

\section{Criteria and Results}

The "normal serum sodium concentration ranges from 135 to $145 \mathrm{mEq}$ per litre' (Cooke and Ottenheimer, 1960). We have adopted the standard of Harrison and Finberg (1959) that any value for serum sodium of $150 \mathrm{mEq} / \mathrm{l}$. or above constitutes hypernatraemia. The range of values for serum sodium in this series was from 118 to $200 \mathrm{mEq} / \mathrm{l}$. The distribution of the measurements is shown in the Figure. It can be seen that $150 \mathrm{mEq} / 1$. is an obvious level at which to separate the cases, the step-wise decrease in numbers being replaced at that point by a sharp increase.

In Table 1 are listed the reports available to us with the percentages of cases in the various groups. The incidence of hyponatraemia (serum sodium of $130 \mathrm{mEq} / \mathrm{l}$. or less) is fairly constant in all the series in which it is listed. There is marked variation, however, in the incidence of hypernatraemia which cannot be satisfactorily explained on the information available. However, in the nine series in which actual figures are given, of a total of 976 infants, 206 or $21 \%$ were hypernatraemic. Our series contains more hypernatraemic cases than the average, but considerably fewer than in two of the reports. We have thus established that hypernatraemic dehydration is as common here as in other areas.

Mortality. Eight of the 100 patients died. In two cases death might be attributed directly to the diarrhoea. One was a mongol who responded poorly to treatment; the second baby died a week after admission and at autopsy was found to have extensive ulcerative colitis. Two other children died several weeks after the initial attack of diarrhoea and at autopsy showed only bronchopneumonia; one of these was also a mongol. The remaining four patients died at intervals of from three to 30 days after the serum electrolyte measurement and all four showed gross cerebral changes at autopsy. The pathological conditions were described as follows: multiple cerebral haemorrhages; multiple areas of cerebral necrosis; cerebral infarction; and multiple areas of cerebral necrosis with calcification. It is a striking fact that three of these four 'cerebral' deaths occurred among the 30 patients with hypernatraemia-indeed they were the only deaths in this group-while only one occurred in the remaining 70 patients. This bears out what must, we think, be accepted as an established fact, that there is an association between hypernatraemia and brain injury.

Contrary to the experience of others, hypernatraemia did not in this series carry a significantly higher mortality than normo- or hyponatraemia, the overall mortality in the former being $10 \%$ and in the latter $7 \%$.

Neurological Disturbances. Clinical evidence of disturbances of the nervous system is most con-

TABLE 1

INCIDENCE OF HYPO- AND HYPERNATRAEMIA IN PREVIOUS REPORTS

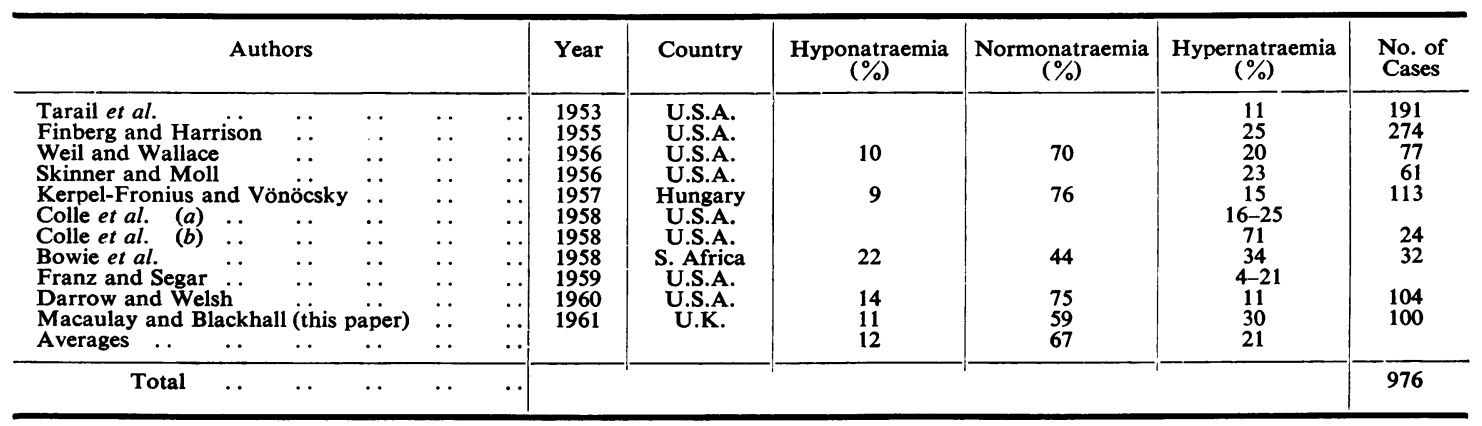


vincingly documented by the occurrence of convulsions and by the frequency with which lumbar puncture was performed. It is reasonable to suppose that a child subjected to lumbar puncture must have had some symptom or sign suggestive of C.N.S. disorder. Convulsions were recorded in 10 of the hypernatraemic group, in nine of them during rehydration, and in seven of the remaining cases, five of these occurring during treatment. Statistically this difference is significant at the $2 \%$ level and almost reaches the $1 \%$ level. The difference between the two groups in the frequency with which lumbar puncture was performed $(14$ or $47 \%$ of the hypernatraemic patients, 17 or $24 \%$ of the remainder) is just significant at the $5 \%$ level. A less reliable, because more subjective, criterion of nervous disorder is recording disturbances of consciousness. These ranged from marked lethargy to deep coma. Since the existence of the syndrome of hypernatraemic dehydration was, we are confident, unsuspected by the observers making the notes any bias in recording this symptom should affect all cases equally. In this case the difference between the two groups is highly significant. Of the hypernatraemic patients $14(47 \%)$ and of the remainder $12(17 \%)$ showed some depression of consciousness $(p<0 \cdot 01)$.

The protein concentration in the cerebrospinal fluid was estimated in 14 samples from hypernatraemic patients and in 15 from other cases. The values ranged very widely in both groups-from 20 to $200 \mathrm{mg} . / 100 \mathrm{ml}$. in the former, from 20 to $500 \mathrm{mg}$. $/ 100 \mathrm{ml}$. in the latter. The average value in the hypernatraemic patients was $70 \mathrm{mg} . / 100 \mathrm{ml}$, and in the others $80 \mathrm{mg} . / 100 \mathrm{ml}$. This latter figure is a little misleading as there were two very high values in this group. If $40 \mathrm{mg}$. $/ 100 \mathrm{ml}$. is taken as the upper limit of normal, there were nine high values in the 14 hypernatraemic samples and six in the 15 from the other patients. This difference, though suggestive, is not significant $(p>0 \cdot 3)$. We have, in other words, failed to confirm that hypernatraemia is regularly associated with raised protein levels in the cerebrospinal fluid.

The incidence of hyperirritability and increased muscle tone could not be determined with any accuracy from the records available since they were infrequently noted in any group.

The follow-up period was, for many patients, too short to permit any evaluation of ultimate neurological status.

There is, in the present series as in those previously reported, definite evidence of an association between hypernatraemia and nervous disturbances. However, it is not a consistent relationship. In the
30 hypernatraemic patients no record of any neurological upset was found in 11 cases. Contrariwise, 22 of the 70 non-hypernatraemic patients had one or more of the symptoms of nervous disorder mentioned in the introductory paragraphs (see also the distribution of cases with nervous disturbances in the Figure). In terms of the numbers at risk there were twice as many patients with neurological disturbances in the hypernatraemic group as in the remainder. A small number of the latter would undoubtedly have been diagnosed on clinical grounds as hypernatraemic, and a considerable number of the former would have been misdiagnosed on the evidence of clinical observation. Since becoming interested in the subject we have made both these mistakes and conclude that the clinical diagnosis of hypernatraemia is unreliable.

TABLE 2

SIGNS OF DEHYDRATION IN CASES WITH WEIGHT LOSS OF $10 \%$ OR MORE

\begin{tabular}{c|c|c}
\hline $\begin{array}{c}\text { Clinical Assessment of } \\
\text { Degree of Dehydration }\end{array}$ & $\begin{array}{c}\text { Hypernatraemic } \\
\text { Cases }\end{array}$ & $\begin{array}{c}\text { Other } \\
\text { Cases }\end{array}$ \\
\hline 0 & 3 & 1 \\
+ & 5 & 6 \\
\hline Total 0 and + & 8 & 7 \\
\hline++++ & 5 & 11 \\
\hline Total ++ and +++ & 1 & 14 \\
\hline
\end{tabular}

$\chi^{2}=1.09 \quad p=0.3$

Signs of Dehydration. As noted in the introduction, the extent of fluid loss in hypernatraemic dehydration is said to be often underestimated because of the lack of the usual signs of dehydration. We have tried to assess this factor in the following way. In some cases, e.g. hospital-acquired infections, the weight before the onset of the diarrhoea was known. In the others the weight charts were used to estimate the gain in weight due to rehydration. From these data the amount of weight lost as the result of the diarrhoea was estimated and expressed as a percentage of the normal weight. Of the 85 patients whose records were complete enough to permit these calculations 35 were estimated to have lost $10 \%$ or more of their body weight, i.e. were moderately severely or very severely dehydrated. The clinical assessment of the degree of dehydration in these cases is shown in Table 2. We are not perturbed by the lack of precision in the terms used in recording the apparent degree of dehydration, nor by the fact that nearly half the cases (15 of the 35 ) were wrongly assessed. The point at issue is whether this mistake is more common when the patient is hypernatraemic. In fact 
the distribution of the cases in this Table does not differ from chance expectancy. Our subsequent experience confirms this finding and we have found it impossible to predict the serum sodium level from the apparent severity of clinical dehydration.

Oedema. Oedema was noted to have occurred in six of the 30 hypernatraemic cases during treatment and in only two of the 70 others. While this is an interesting observation, and indeed a 'significant' one in the statistical sense (the probability of it being a chance occurrence is very nearly one in 100), it hardly bears out the contention that the development of oedema is characteristic of the hypernatraemic state. Mild degrees of oedema may have escaped notice and close observation might yield higher figures. We find it hard to believe, however, that appreciable oedema is at all common in hypernatraemic infants.

\section{Aetiological Factors}

We have reviewed our cases to try to determine what factors might have resulted in 30 of them becoming hypernatraemic. In order to permit statistical evaluation we have given numerical values to the data wherever possible. The notes are not equally informative in all cases and we show the numbers of cases in which the data were considered satisfactory either in the Tables or in brackets after each sub-heading.

No difference between the hypernatraemic patients and the others was found in respect of the following items and we shall not give detailed figures: sex, age, premature birth, previous history of nervous or renal disease, seasonal incidence (the absence of any climatic influence is shown by the numbers with hypernatraemia occurring in successive sixmonthly periods, viz. five, five, four, five, six), frequency of vomiting, character of stools (whether merely 'loose' or described as 'watery'), or number of stools per day. The amount of sodium in the diet before the electrolyte estimation was not a factor in the development of hypernatraemia, but in view of the importance attached to this by some American writers, we shall consider it in some detail.

Sodium Intake. The object of this analysis was to see if, as has been claimed (Finberg, 1958), excessive administration of salt-containing fluids was responsible for a significant number of patients becoming hypernatraemic. This is of obvious importance, since it might lead to recommendations which could result in reducing the number of infants with this complication of diarrhoea.
TABLE 3

ESTIMATED SODIUM INTAKE

\begin{tabular}{|c|c|c|c|}
\hline \multirow{2}{*}{$\begin{array}{c}\text { Sodium } \\
\text { Intake } \\
\text { (mEq/kg./day) }\end{array}$} & \multicolumn{3}{|c|}{ Group } \\
\hline & $\begin{array}{c}\text { Hypo- } \\
\text { natraemia }\end{array}$ & $\begin{array}{c}\text { Normo- } \\
\text { natraemia }\end{array}$ & $\begin{array}{c}\text { Hyper- } \\
\text { natraemia }\end{array}$ \\
\hline $\begin{array}{l}<5 \\
5-10 \\
>10\end{array}$ & $\begin{array}{l}5 \\
5\end{array}$ & $\begin{array}{r}23 \\
17 \\
3\end{array}$ & $\begin{array}{r}16 \\
9 \\
2\end{array}$ \\
\hline Totals $\ldots$ & 10 & 43 & 27 \\
\hline
\end{tabular}

Where the information available made it feasible, we have attempted to assess the sodium intake of the patients in the 48 hours before the electrolyte estimation was done. Very few of the patients were given supplementary salt-containing fluids before admission. This represents a marked difference from experience in America (Colle, Ayoub and Raile, 1958; Franz and Segar, 1959; Harrison and Finberg, 1959) where commercial electrolyte solutions are extensively used in the home treatment of diarrhoea. These estimates cannot, obviously, be very precise, but in 80 of the 100 cases there were sufficient data to justify analysis. We have calculated the amount of sodium in the feeds offered to the infants, ignoring vomiting and anorexia as sources of error. This means that our estimates are of the greatest amounts of sodium likely to have been ingested. In many cases, because of vomiting and anorexia, the actual intakes were much less than those calculated. The sodium content of various feeds was directly measured by having random feeds, made up in the hospital milk-room, analysed in the laboratory. The figures are given in terms of $\mathrm{mEq}$ sodium $/ \mathrm{kg}$. hydrated body weight/day. This makes possible comparison of babies of different weights and it also indicates the importance of the actual load of sodium given. There is some evidence that 'the ceiling for renal excretion of sodium may be in the range of 5 to $10 \mathrm{mEq}$ per kilogram of body weight per day' (Skinner and Moll, 1956). Darrow and Welsh (1960) report that quantities equivalent to $7.5 \mathrm{mEq} / \mathrm{kg}$./day are well tolerated by infants not suffering from dehydration. It may be assumed that intakes of up to $5 \mathrm{mEq} / \mathrm{kg}$./day are normal (e.g. an infant fed on a half-cream dried milk at the rate of $2 \frac{1}{2} \mathrm{oz}$./lb./day will ingest approximately $4 \mathrm{mEq} / \mathrm{kg}$./day). Intakes of over $10 \mathrm{mEq} / \mathrm{kg}$./day are almost certainly excessive. The intermediate amounts, 5 to $10 \mathrm{mEq} / \mathrm{kg}$./day, would probably be tolerated without difficulty by a healthy infant, but might be excessive for an ill one.

The results of our analysis are given in Table 3. Only five infants received amounts of sodium in the frankly excessive range (four of them had been 
rather enthusiastically rehydrated with intravenous fluids before the electrolytes were measured) and of these only two showed hypernatraemia. More than half the hypernatraemic group had been offered, and had not necessarily ingested, quite normal amounts of sodium. In the intermediate group the distribution of cases is almost exactly the same as in the series as a whole, viz. $29 \%$ hypernatraemic. It seems legitimate to conclude that very few of our patients had been overloaded with sodium and that dietary sodium played an insignificant part in determining the serum sodium levels.

The respects in which the hypernatraemic cases differed from the others were: severity of anorexia, duration of diarrhoea, temperature and respiratory rate.

Anorexia. The severity of anorexia has been graded as $\mathbf{0}$ (none), + (mild, 'reluctant to feed') and ++ (severe, 'refused all feeds'). The results of the analysis are shown in Table 4 . The difference between the groups is highly significant $-60 \%$ of the hypernatraemic group having had severe anorexia compared to $24 \%$ of the others.

TABLE 4

SEVERITY OF ANOREXIA IN HYPERNATRAEMIC AND OTHER PATIENTS

\begin{tabular}{c|c|c|c}
\hline $\begin{array}{c}\text { Severity } \\
\text { of } \\
\text { Anorexia }\end{array}$ & \multicolumn{2}{|c|}{ Group } & Totals \\
\cline { 2 - 3 } & Hypernatraemic & Others & \\
\hline 0 or + & 10 & 45 & 55 \\
++ & 16 & 14 & 30 \\
\hline Totals & 26 & 59 & 85 \\
\hline$\chi^{2}=9.7 \quad p<0.01$ &
\end{tabular}

Duration of Diarrhoea (94 cases). The mean duration of diarrhoea before admission, or before blood examination in those developing diarrhoea in hospital, was 3.4 days for the hypernatraemic group and 5.7 days for the others. This is a significant difference $(t=2.16 ; 0.05>p>0.02)$. None of the patients in the first group had been ill for longer than seven days, and several for only a few hours; whereas in the latter groups 18 out of 68 had histories longer than a week.

Temperature (100 cases). The highest temperature recorded on the day of admission (or on the day of electrolyte estimation, if these were different) had been taken as an index of the severity of fever. In most cases, but not in all, these were the highest temperatures recorded during the child's stay in hospital. The average temperatures, and the standard deviations, in the groups were: hypernatraemic cases $100 \cdot 8^{\circ} \mathrm{F} . \pm 2 \cdot 6$; remainder $98 \cdot 6^{\circ}$ F. \pm 1.9 . This difference of $2 \cdot 2^{\circ} \mathrm{F}$. between the means is nearly five times as great as its standard error and is thus highly significant. Nearly half the hypernatraemic cases had fever of $101^{\circ} \mathrm{F}$. or more (14 out of 30), whereas only one in 10 of the remaining cases (seven out of 70) showed such a response.

Respiratory Rate. The respiratory rate at the time of recording the temperature used in the previous section has been tabulated in the same way. Unfortunately, owing to a change in the type of chart used in the wards during the period of this survey, these figures are available for only 46 patients. There is no reason to believe that this has resulted in any bias in the figures: the proportions in the various groups do not differ appreciably from those in the series as a whole: 16 of the 46 were hypernatraemic. In most cases, of course, the respiratory rate paralleled the temperature, but in a small number there was a disproportionate hyperpnoea. The average rates in the groups (with their standard deviations) were as follows: hypernatraemic cases $49 / \mathrm{min}$. $\pm 13 \cdot 8$; others $34 / \mathrm{min}$. \pm 5.9. This is aiso a very highly significant difference $(\mathrm{t}=4.67 ; \mathrm{p}<0.001)$. Of the hypernatraemic cases $75 \%$ (12 out of 16 ) had respiratory rates of $40 / \mathrm{min}$. or above, while only one in 10 (three out of 30 ) of the others had so marked a tachypnoea.

\section{Discussion}

In this series of 100 infants with gastro-enteritis hyperosmolarity of the plasma, as measured by serum sodium levels, occurred in nearly one-third of the patients. In one-third of these there were no characteristic clinical features to differentiate them from other cases of infantile diarrhoea. In the other two-thirds nervous disorders of varying severity, from lethargy to extensive cerebral necrosis, were encountered. This incidence is similar to that reported by other writers (Finberg and Harrison, 1955). On the other hand about $30 \%$ of patients without hypernatraemia demonstrated neurological disturbances of the same type and severity. We have been unable to confirm that hypernatraemic dehydration is regularly associated with increased protein levels in the C.S.F. or that oedema is a frequent occurrence in this condition. We have not found the clinical assessment of the severity of dehydration a reliable index of the presence or absence of hypernatraemia.

The infants with hypernatraemia differed from the others in the following respects: incidence of 
cerebral disturbances, short duration of diarrhoea, frequency of fever and tachypnoea and severity of anorexia.

This account agrees substantially with that given by Bowie, McKenzie and Hansen (1958) from which we quote: 'The clinical recognition of hyperosmolarity in infantile gastro-enteritis is difficult ... It is our impression that those showing the hyperosmolar state had a short history of explosive diarrhoea and vomiting ... Where gastro-enteritis is associated with hyperpyrexia, hyperventilation or severely curtailed fluid intake the possibility of hyperosmolarity developing is considerably enhanced.'

The pathogenesis of the condition does not seem to be particularly mysterious. It is known (Weil and Wallace, 1956) that diarrhoeal stools are hypotonic so that profuse diarrhoea will, in the absence of any compensating mechanism, lead to a greater deficit of water than of electrolyte, i.e. to a condition of hypertonicity. The two possible compensating mechanisms available are (1) an increase in the amount of water ingested to balance the losses and (2) differential excretion of electrolytes and water by the kidneys resulting in the secretion of a concentrated urine and maintenance of the tonicity of the body fluids. In infants neither of these mechanisms is as effective as in adults: (1) Infants are dependent on others for their fluid intake and the need for increased amounts of fluid may not be appreciated by the attendants. Even with an appreciation of these needs, severe anorexia may make it impossible to get enough fluid into the child. This was an important factor in our patients. (2) Under the stress of dehydration, and perhaps aggravated by losses of electrolytes, especially potassium, in the stools, renal function becomes impaired and the infantile kidneys become incapable of excreting a concentrated urine. The few studies reported on hypernatraemic infants (Weil and Wallace, 1956) confirm the existence of this type of dysfunction, manifested by the failure to excrete as concentrated a urine as would have been expected. This temporary renal failure is made worse by further losses of water from the skin and lungs through fever and hyperventilation. It may also contribute to further dehydration since the kidneys will require a greater volume of water for the excretion of solutes than if they are functioning normally.

In any event the development of hyperosmolarity is determined by disproportionate losses of water relative to sodium. This can occur without diarrhoea, and, in fact, the highest level of serum sodium encountered in any case in the two years and nine months of our survey was $235 \mathrm{mEq} / 1$. (duplicate estimation) in an infant with congenital pyloric stenosis who was constipated but dehydrated and wasted from persistent vomiting.

With regard to treatment, recommendations have varied from elaborate schedules (Skinner and Moll, 1956) to statements that 'Patients with hypernatraemia received the usual fluid therapy used in other cases of diarrhoea' (Darrow and Welsh, 1960). In general it is agreed that repair solutions should be hypotonic and that the replacement of deficits should be spread over 48 hours. Our custom is to use 'fifth-normal saline in glucose water', i.e. a solution containing $0 \cdot 18 \% \mathrm{NaCl}$ and $4 \cdot 3 \%$ glucose, and half-strength plasma, as repair solutions in all cases of diarrhoea requiring intravenous fluids. In the presence of hypernatraemia, we substitute varying amounts of $5 \%$ glucose-water for the 'fifth-normal saline', allow rather more than usual for the repair of existing deficits and aim at a somewhat less rapid correction of these deficits than in the ordinary case. Serum sodium levels vary so much, as do other features of these cases, that we think it unwise to lay down definite rules.

\section{Summary}

A brief review of the recent literature on the occurrence of hypernatraemic dehydration in infantile gastro-enteritis is followed by short accounts of two patients with this condition.

Analysis of the case records of 100 consecutive infants with gastro-enteritis whose serum electrolytes were determined reveals the following:

(1) An incidence of hypernatraemia similar to that reported from other centres;

(2) An association between hypernatraemia and the occurrence of neurological disturbances, similar to that reported by others, but neither as close nor as consistent as sometımes claimed;

(3) The importance of anorexia, fever and hyperventilation in the genesis of the condition.

Pathogenesis and treatment are briefly discussed.

\section{REFERENCES}

Bowie, M. D., McKenzie, D. and Hansen, J. D. L. (1958). Hyperosmolarity in infantile gastro-enteritis. S. Afr. med. J., 32, 322.

Colle, E., Ayoub, E. and Raile, R. (1958). Hypertonic dehydration (hypernatremia): The role of feedings high in solutes. Pediatrics, 22, 5 .

Cooke, R. E. (1959). Parenteral fluid therapy. In Textbook of Pediatrics, ed. Nelson, W. E., 7th ed., pp. 183-98. Saunders, Philadelphia and London.

Philadelphia and London. (1960). Clinical and experimental interrelations of sodium and the central nervous system. In interrelations of sodium and the central nervous system. In
Advances in Pediatrics, ed. Levine, S. Z., Vol. XI, pp. 81-145. The Year Book Publishers, Chicago.

Darrow, D. C. and Welsh, J. S. (1960). Recent experience in the treatment of diarrhea in infants. J. Pediat., 56, 204. 
Finberg, L. (1958). The possible role of the physician in causing hypernatremia in infants dehydrated from diarrhea. Pediatrics, 22, 2.

- and Harrison, H. E. (1955). Hypernatremia in infants. An evaluation of the clinical and biochemical findings accompanying this state. Ibid., 16, 1.

Franz, M. N. and Segar, W. E. (1959). The association of various factors and hypernatremic diarrheal dehydration. Amer, $J$. Dis. Child., 97, 298.

Harrison, H. E. and Finberg, L. (1959). Hypernatremic dehydration Pediat. Clin. N. Amer., Vol. 6, No. 1, p. 193.
Kerpel-Fronius, E. and Vönöczky, J. (1957). Significance of changes in the tonicity of body fluids in infantile diarrheal dehydration. Ann. Paediat. Fenn., 3, 403.

Rapoport, S. (1947). Hyperosmolarity and hyperelectrolytemia in patholog

Skinner, A. L. and Moll, F. C. (1956). Hypernatremia accompanying infant diarrhea. Ibid., 92, 562 .

Tarail, R., Bass, L. W. and Runco, A. S. (1953). The frequency and nature of hypertonicity of the body fluids in infantile diarrhea. Ibid., 86, 658.

Weil. W. B, and Wallace, W. M. (1956). Hypertonic dehydration in infancy. Pediatrics, 17, 171. 